\title{
Kinematics and Ages of UV Ceti Stars
}

\author{
A. Poveda, C. Allen, M.A. Herrera ${ }^{\star}$ \\ Instituto de Astronomia, Universidad Nacional Autonoma de Mexico, Mexico
}

\begin{abstract}
The kinematic properties of 93 UV Ceti stars of the solar neighborhood are studied, based on a list of flares within $25 \mathrm{pc}$ of the Sun $\left(\pi \geq 0^{\prime \prime} 04\right)$. With updated values for their distances, proper motions and radial velocities (Gliese \& Jahreiss 1991) space velocity dispersions are calculated for these stars. It is found that the total velocity dispersion of the flare stars $\left(\sigma=30 \pm 3 \mathrm{~km} \mathrm{~s}^{-1}\right)$ is similar to that of the F5 V stars from the same catalogue, for which the conventionally estimated mean age is about $3 \cdot 10^{9}$ years. A number of flare stars are identified as members of the Hyades, Sirius or Pleiades groups. The velocity dispersions found for the nearby flare stars, as well as their scale height and the membership of some of them to young kinematic groups, indicate that they belong to the young disk population. A small number (7) of UV Ceti stars have kinematics corresponding to the thick disk or halo population. Their long-lived chromospheric activity is interpreted as due to coalescence of old contact binaries. The question of the age of Proxima Centauri is examined in the context of our results, and found to be compatible with the ages of $\alpha$ Centauri A and B.
\end{abstract}

\section{Introduction}

It is well known that pre-main sequence late type stars in young clusters and associations exhibit eruptive behavior similar to that of the classic UV Cet stars in the solar vicinity, as was found for the first time by Haro \& Morgan (1953) in Orion, and by Haro extensively in Orion, NGC 2264, the Pleiades, the Hyades, etc. (Haro 1968, and references therein). Because the physical and environmental characteristics of such groups are clearly very different from those of the solar vicinity, Haro called "flash stars" the flare-like stars found in clusters and associations.

From the work by Haro and Chavira in Tonantzintla, Rosino in Asiago, and Ambartsumian and his collaborators in Byurakan (see Haro 1968, Gershberg 1971 and Gurzadyan 1980 for extensive references), a number of statistical regularities emerged both among the flash stars and also among the UV Cet stars in

\footnotetext{
* Also at Programa Universitario de Investigacion y Desarrollo Espacial (PUIDE) UNAM
} 
the solar vicinity. One of the regularities of particular interest to us here involves the correlation between the age of a cluster (or association) and the spectral type of the earliest flares in it: the older the cluster, the later its flare stars, ranging from $\mathrm{K} 0-\mathrm{K} 2$ for the flash stars in Orion to $\mathrm{M} 3-\mathrm{M} 5$ in the Hyades, the latter being similar to the spectra of the UV Cet stars in the solar vicinity. This similarity led Haro to an estimate of about $10^{9}$ years for the ages of the oldest UV Cet stars.

Boesgard \& Hagen (1974) pointed out an inconsistency between the ages found for components A and B of the $\alpha$ Cen system and Haro \& Chavira's mean age for UV Cet stars. Specifically, by means of the intensities of the $H$ and $\mathrm{K}$ emission lines, the rotation periods, and the intensity of the lithium lines, Boesgard \& Hagen estimated an age of about $3-4 \cdot 10^{9}$ years for $\alpha$ Cen $A$ and $B$, while for Proxima, a well-known flare star and a convincing member of the system, an expected age of only $4 \cdot 10^{8}$ years followed from Haro \& Chavira's scheme. The question of the age of the flare stars was further discussed by Kunkel (1975) and others, who pointed out evidence indicating that faint UV Cet stars flare throughout the life of the Galaxy.

With the motivation provided by the preceding comments, we study in the present paper the kinematic properties of the nearby UV Cet stars, and we compare them with those of other types of stars in the solar vicinity, whose nuclear ages are well-known. We note that the velocity dispersion of stars in the galactic disk is a monotonically increasing function of age (Wielen 1977) and therefore can be used, as is done here, to date a group of stars. To this end, we use modern data on nearby UV Cet stars, mostly from Gliese \& Jahreiss' (1991) catalogue of stars closer than $25 \mathrm{pc}$.

\section{Kinematic properties of nearby UV Cet stars}

A working list of stars nearer than 25 pc and exhibiting reasonably certain flare activity has been compiled. The basis for this list is the catalogue provided by Pettersen (1991), which contains all known flare stars with a trigonometric parallax $\pi \geq 0$ ".04 and a time-resolved record (usually a photometric light curve in optical or X-ray wavelengths) of one or more flares. The lists of Gurzadyan (1980), Kunkel (1975) and Rodono (1978) and the notes to the Gliese (1969) catalogue were also consulted, but we only added stars from these lists to ours when the star was nearer than $25 \mathrm{pc}$ and there seemed to be no doubt about its flare activity. Information on distances, proper motions and radial velocities for these systems was checked and updated using the data provided by Gliese \& Jahreiss (1991). In such a way, a list of 86 systems containing a total of 108 stars with reliably reported flare activity was obtained. However, only 74 of these systems, with a total of 93 stars, have sufficient information to allow the calculation of their space velocities.

A small number of flare stars exhibit very discordant kinematics (high space velocities), clearly implying that they do not belong to the normal disk population. The most extreme example is Groombridge $1830 \mathrm{~B}$, whose visual companion 
Table 1. Velocity dispersions of nearby UV Ceti and main sequence F stars

\begin{tabular}{lcccc}
\hline & $\begin{array}{c}\sigma_{U} \\
(\mathrm{~km} / \mathrm{s})\end{array}$ & $\begin{array}{c}\sigma_{V} \\
\mathrm{~km} / \mathrm{s})\end{array}$ & $\begin{array}{c}\sigma_{W} \\
(\mathrm{~km} / \mathrm{s})\end{array}$ & $\begin{array}{c}\sigma \\
(\mathrm{km} / \mathrm{s})\end{array}$ \\
\hline All 86 flare stars & 27.4 & 12.5 & 14.7 & 33.5 \\
43 brightest flare stars & 26.7 & 13.8 & 14.8 & 33.5 \\
43 faintest flare stars & 27.8 & 11.6 & 13.7 & 33.1 \\
33 flare stars with $M_{V} \leq 13, d \leq 13^{6} \mathrm{pc}$ & 22.7 & 10.0 & 10.4 & 26.9 \\
56 F4+F5+F6 stars & 25.7 & 12.5 & 15.1 & 32.3 \\
\hline
\end{tabular}

is spectroscopically classified as a subdwarf; this system has a heliocentric space velocity of $304 \mathrm{~km} \mathrm{~s}^{-1}$. Other examples, although less extreme, are Gl 412B, Gl 424 and Gl 781, photometrically classified as metal-poor, and Gl 166C, Gl 630.1 and Gl 866.

Velocity dispersions were calculated by the method of percentiles for the 86 flare stars with reliable space velocities, after eliminating the 7 discordant ones (see Table 1). This study shows that there are no significant differences in the velocity dispersions of the faint flare stars compared to the brighter ones. A subgroup of the working list, namely systems closer than $13 \mathrm{pc}$ and with visual magnitudes brighter than $M_{V}=13$, which should be less affected by incompleteness and kinematical bias than the entire sample, yields a total velocity dispersion of $\sigma=27 \pm 3 \mathrm{~km} \mathrm{~s}^{-1}$. This value is somewhat smaller than the velocity dispersion for the whole list, namely $\sigma=34 \pm 3 \mathrm{~km} \mathrm{~s}^{-1}$, pointing to the presence of small kinematical bias in favor of the fainter and remote flare stars. Therefore, we may adopt $\sigma=30 \pm 3 \mathrm{~km} \mathrm{~s}^{-1}$ as a reliable estimate of the velocity dispersion of the UV Cet stars. A comparison of the velocity dispersion of those flares that are members of double and multiple systems, with those that are single, shows no significant difference.

\section{Discussion and conclusions}

The kinematic properties of the bulk of the UV Cet stars most closely resemble those of main sequence stars of types F4 - F6 in the solar vicinity. For these stars, the estimated mean nuclear ages are about $3 \cdot 10^{9}$ years (Iben 1967). Assuming that there has been on the average a constant rate of star formation in the galactic disk over the last $8 \cdot 10^{9}$ years, we conclude that the age of individual UV Ceti stars may span the whole interval from 0 to $6 \cdot 10^{9}$ years, or even from 0 to $8 \cdot 10^{9}$ years, when we consider the longer timescales obtained by Maeder \& Maynet (1988) for models with convective overshooting. In other words, we may find normal UV Cet stars as old as the Sun (or somewhat older), a fact which would resolve the inconsistency pointed out by Boesgard \& Hagen (1974) for the $\alpha$ Cen system.

There remains a small group of apparently single, high velocity - and hence presumably very old - flare stars whose persistent activity remains unexplained. 
One conjecture worth exploring involves the occasional coalescence of the components of old contact binaries, which would produce in most cases a fully convective single star with high rotational velocity; such a star would become chromospherically active, with $\mathrm{H} \alpha$ emission, flare activity, etc., but with kinematic properties that would advertise its membership to an old population. Because of the loss of angular momentum through flare activity and stellar winds the fate of these short-period binaries is to coalesce into a rejuvenated rapidly rotating single star. In fact, van't Veer \& Maceroni (1989) have found the time-scale for coalescence of short period $(P<4 \mathrm{~d})$ late type main sequence binaries to be $\approx 7.5 \cdot 10^{8}$ years. Some of the high velocity stars of our list may have this origin.

The supercluster membership of a significant fraction of the flare stars in the solar vicinity (Hyades: 10, Pleiades-Perseus-Taurus: 4; Sirius: 4; Wolf 630: 3) suggests that UV Cet stars may indeed be identified with the oldest members of the flash star population once belonging to galactic clusters that have long ago been disrupted, and whose members have populated the general galactic field.

Acknowledgements: This work is part of a more comprehensive investigation submitted to A\&A. We wish to acknowledge support of the University Space Program (PUIDE). Thanks are due to James L. Green of World Data Center, and to Wayne H. Warren, Jr., of the Astronomical Data Center for providing the CD-ROM of selected astronomical catalogues, and to G. Cordero, C. Lavalley and A. Nigoche for computing assistance. This research has made use of the Simbad database, operated at CDS, Strasbourg, France.

\section{References}

Boesgard A.M., Hagen W., 1974, ApJ 189, 85

Gershberg, R.E. 1971, Flares of red dwarf stars, Crimean Astroph. Obs. Translated by D.J. Mullan, Armagh Obs. North Ireland

Gliese W. 1969, Veröffentl. Astron. Rechen-Inst. Heidelberg, 22

Gliese W., Jahreiss H., 1991, in L.E. Brotzman, S.E. Gessner: Selected Astronomical Catalogs, Volume I, National Space Science Center, NASA GSFC

Gurzadyan G.A., 1980, in Flare Stars, Y. Terzian (ed.), Pergamon, Oxford

Haro G., 1968, in Stars and Stellar Systems, Vol. 7, Nebulae and Interstellar Matter, B.M. Middlehurst \& L.H. Aller (eds.), University of Chicago Press, Chicago, p. 141

Haro G., Chavira E., 1966, in Vistas in Astronomy, Vol. 8, A. Beer (ed.), Pergamon, Oxford, p. 89

Haro G., Morgan W.W., 1953, ApJ 118, 16

Iben I., Jr. 1967, ARAA 5, 571

Kunkel W.E., 1975, in Variable Stars and Stellar Evolution, V.E. Sherwood \& L. Plaut (eds.), Reidel, Dordrecht, p. 15

Maeder A., Meynet G., 1988, A\&AS 76, 411

Pettersen B.R., 1991, Mem. Soc. Astr. Ital., 62, 217

Rodonó M., 1978, A\&A 66, 175

van't Veer F., Maceroni C., 1989, A\&A 220, 128

Wielen R., 1977, A\&A 60, 263 